\title{
Bell's theorem without inequalities and without probabilities for two observers
}

\author{
Adán Cabello* \\ Departamento de Física Aplicada II, Universidad de Sevilla, 41012 Sevilla, Spain
}

(October 30, 2018)

A proof of Bell's theorem using two maximally entangled states of two qubits is presented. It exhibits a similar logical structure to Hardy's argument of "nonlocality without inequalities". However, it works for $100 \%$ of the runs of a certain experiment. Therefore, it can also be viewed as a Greenberger-Horne-Zeilinger-like proof involving only two spacelike separated regions.

PACS numbers: 03.65.Ud, 03.67.-a, 42.50.-p

Bell's theorem [1] states that one cannot in general reproduce the results of quantum theory with a classical, deterministic local model. Hardy's argument of "nonlocality without inequalities" [2] is considered "the best version of Bell's theorem" [3]. Curiously enough, while in the original proof of Bell's theorem using inequalities the maximum discrepancy with local models occurs for maximally entangled states [- (also known as "Bell states" [5]), Hardy's proof is not valid for maximally entangled states. It works only for entangled but non-maximally entangled states (also known as "Hardy states" [6]). This curious feature has led to several attempts and suggestions to develop a Hardy-like argument for maximally entangled states of two qubits. However, so far none of these proposals has worked [7]. In addition, Hardy's argument works only for $9 \%$ of the runs of a certain experiment. On the other hand, the proof of Bell's theorem by Greenberger, Horne, and Zeilinger (GHZ) 8 11] works for $100 \%$ of the runs, but requires three observers (instead of two, as in Hardy's proof).

In this paper, I introduce a Hardy-like proof which works for $100 \%$ of the runs of an experiment. It requires only two observers and is based on maximally entangled states of two qubits. This proof is more complicated than the original by Hardy, since it involves two copies of the maximally entangled state (instead of just one), but it preserves the logical structure of the original argument. The proof is inspired in Hardy's argument [2] (see also 3.6. 12, 13]) and in a proof of the Kochen-Specker theorem 114] by Peres [15] and Mermin [16] (see also [17 20]).

A qubit is a quantum two-level system like, for instance, the spin state of a spin- $\frac{1}{2}$ particle. Consider a source of two qubits in the singlet state

${ }^{*}$ Electronic address: adan@cica.es

$$
\left|\psi^{-}\right\rangle_{i j}=\frac{1}{\sqrt{2}}\left(|01\rangle_{i j}-|10\rangle_{i j}\right),
$$

where $|01\rangle_{i j}=|0\rangle_{i} \otimes|1\rangle_{j}$, and where $\sigma_{z}|0\rangle=|0\rangle$ and $\sigma_{z}|1\rangle=-|1\rangle, \sigma_{z}$ and $\sigma_{x}$ being the Pauli spin matrices. Suppose the source emits two singlets so that the initial state is

$$
|\psi\rangle_{1234}=\left|\psi^{-}\right\rangle_{12} \otimes\left|\psi^{-}\right\rangle_{34}
$$

The scenario for the proof is the following: particle 1 moves away from particle 2, and particle 3 moves away from particle 4 . At a given time, an observer, Alice, has access to particles 1 and 3, while in a spacelike separated region a second observer, Bob, has access to particles 2 and 4 .

I will use the following notation: For particles 1 and 3 (Alice's particles) $A_{i}=\sigma_{z i}$ and $a_{i}=\sigma_{x i}$; for particles 2 and 4 (Bob's particles) $B_{j}=\sigma_{z j}$ and $b_{j}=\sigma_{x j}$. On the other hand, the product of two letters represents the product of the corresponding operators; for instance, $A_{1} A_{3}=\sigma_{z 1} \otimes \sigma_{z 3}$. The results of the measurements of all these operators can be either -1 or +1 .

Using this notation, the state (2) has the following properties:

$$
\begin{aligned}
P_{\psi}\left(A_{1}=B_{2}\right) & =0, \\
P_{\psi}\left(a_{1}=b_{2}\right) & =0, \\
P_{\psi}\left(A_{3}=B_{4}\right) & =0, \\
P_{\psi}\left(a_{3}=b_{4}\right) & =0,
\end{aligned}
$$

and

$$
\begin{aligned}
P_{\psi}\left(B_{2}=B_{4} \mid A_{1} A_{3}=+1\right) & =1, \\
P_{\psi}\left(b_{2}=b_{4} \mid a_{1} a_{3}=+1\right) & =1, \\
P_{\psi}\left(A_{1}=a_{3} \mid B_{2} b_{4}=+1\right) & =1 \\
P_{\psi}\left(a_{1}=-A_{3} \mid b_{2} B_{4}=-1\right) & =1
\end{aligned}
$$

and

$$
\begin{aligned}
P_{\psi}\left(A_{1} A_{3}\right. & =+1, a_{1} a_{3}=+1, \\
B_{2} b_{4} & \left.=+1, \quad b_{2} B_{4}=-1\right)=\frac{1}{8},
\end{aligned}
$$

where, $P_{\psi}\left(A_{1}=B_{2}\right)$ is the probability of $A_{1}$ and $B_{2}$ giving the same result, and $P_{\psi}\left(B_{2}=B_{4} \mid A_{1} A_{3}=+1\right)$ is the conditional probability of $B_{2}$ and $B_{4}$ having the same result given that the result of $A_{1} A_{3}$ is +1 . Properties (33)(11) will be proved later. Now let us focus our attention 
on the logical argument of proving Bell's theorem that can be deduced from them.

Properties (3)-(6) allow us to establish that there exist "elements of reality", as defined by Einstein, Podolsky, and Rosen (EPR) 21], corresponding to $A_{1}, B_{2}, a_{1}, b_{2}$, $A_{3}, B_{4}, a_{3}$, and $b_{4}$. According to EPR, "If, without in any way disturbing a system, we can predict with certainty (i.e., with probability equal to unity) the value of a physical quantity, then there exists an element of physical reality corresponding to this physical quantity" [21. Property (3) tells us that if Alice (Bob) measures $A_{1}$ on particle 1 ( $B_{2}$ on particle 2$)$, then she (he), without in any way disturbing particle 2 (particle 1 ), which is assumed to be in a distant spacelike separated region, can predict with certainty the result of $B_{2}\left(A_{1}\right)$. Therefore, there exists an element of reality corresponding to $B_{2}$ $\left(A_{1}\right)$. This implies that its value was present before Alice's (Bob's) measurement, and thus does not depend on Alice's (Bob's) choice of experiment. Identical arguments based on properties (4)-(6) lead us to establish that there exist elements of reality corresponding to $a_{1}, b_{2}, A_{3}, B_{4}$, $a_{3}$, and $b_{4}$.

On the other hand, property (7) tells us that, when Alice measures $A_{1} A_{3}$ on particles 1 and 3 , and finds the result +1 , then she, without in any way disturbing particles 2 and 4, which are assumed to be in a distant spacelike separated region, can predict with certainty that if Bob measures $B_{2}$ and $B_{4}$, he will find the same result for both. Since $B_{2}$ and $B_{4}$ are elements of reality, they were determined before Alice's measurement and thus cannot depend on Alice's choice of experiment. Properties (8)(10) lead to similar arguments.

The four physical magnitudes $A_{1} A_{3}, a_{1} a_{3}, B_{2} b_{4}$, and $b_{2} B_{4}$ are represented by commutative operators and thus they can be jointly measured on the same system. Indeed, according to property (11), the four conditions appearing in (7)-(10) (i.e., $A_{1} A_{3}=+1, a_{1} a_{3}=+1$, $B_{2} b_{4}=+1, b_{2} B_{4}=-1$ ) can occur simultaneously (they occur in average in $\frac{1}{8}$ of the runs of the experiment in which Alice measures $A_{1} A_{3}$ and $a_{1} a_{3}$, while Bob measures $B_{2} b_{4}$ and $\left.b_{2} B_{4}\right)$. Therefore, for those events in which Alice finds $A_{1} A_{3}=+1$ and $a_{1} a_{3}=+1$, and Bob finds $B_{2} b_{4}=+1$ and $b_{2} B_{4}=-1$, the values of the elements of reality must satisfy the following relations:

$$
\begin{aligned}
v\left(B_{2}\right) & =v\left(B_{4}\right), \\
v\left(b_{2}\right) & =v\left(b_{4}\right), \\
v\left(A_{1}\right) & =v\left(a_{3}\right), \\
v\left(a_{1}\right) & =-v\left(A_{3}\right) .
\end{aligned}
$$

However, any assignment of values, either -1 or +1 , that satisfies (12)-(15), would be in contradiction with one of the properties (3)-(6). To prove this, let us suppose that the four properties (3)-(6) were satisfied. Then, the values of the elements of reality would satisfy the following relations:

$$
\begin{gathered}
v\left(A_{1}\right)=-v\left(B_{2}\right), \\
v\left(a_{1}\right)=-v\left(b_{2}\right), \\
v\left(A_{3}\right)=-v\left(B_{4}\right), \\
v\left(a_{3}\right)=-v\left(b_{4}\right) .
\end{gathered}
$$

However, the eight Eqs. (12)-(19) cannot be satisfied simultaneously because when we take the product of all of them, the result is

$$
v\left(B_{2}\right) v\left(b_{2}\right) v\left(A_{3}\right) v\left(a_{3}\right)=-v\left(B_{2}\right) v\left(b_{2}\right) v\left(A_{3}\right) v\left(a_{3}\right) .
$$

Eq. (20) has no solution because $B_{2}, b_{2}, A_{3}$, and $a_{3}$ can take only the values -1 and +1 . We therefore conclude that the predictions of quantum theory for these events cannot be reproduced with any classical local model based on EPR criterion of elements of reality.

As a close examination will reveal, a similar contradiction can be found every time the product of the results of (Alice's measurements) $A_{1} A_{3}, a_{1} a_{3}$ (and of Bob's measurements), $B_{2} b_{4}$, and $b_{2} B_{4}$ is -1 , and there is no contradiction if the product is +1 . In principle, there are 16 possible outcomes; in eight of them, the product is +1 , and in the other eight, the product is -1 . It would therefore be interesting to calculate the probability of occurrence of each of the 16 possible outcomes. The results of these calculations (which will be explained in more detail later) are in Table I. Surprisingly, as a scrutiny of Table I shows, only those events in which the product of the results of $A_{1} A_{3}, a_{1} a_{3}, B_{2} b_{4}$, and $b_{2} B_{4}$ is -1 have a nonzero probability to occur. That is, only those results that cannot be described with local models occur. Therefore, according to the predictions of quantum mechanics, all the events of the experiment in which Alice measures $A_{1} A_{3}$ and $a_{1} a_{3}$, while Bob measures $B_{2} b_{4}$ and $b_{2} B_{4}$, cannot be reproduced with any local model.

This argument exhibits a similar logical structure to Hardy's. Both require a series of experiments to establish that all the outcomes that never happen (or always happen) are indeed never (or always) found. In our case, these experiments are those to test quantum predictions (3)-(10). Having done that, and having drawn inferences based on EPR criterion, both arguments come to a crucial experiment. In Hardy's crucial experiment a maximum of $9 \%$ of the runs [2,3,13 (or almost $50 \%$ in its "ladder" version [22,23) yield results that are in contradiction with the EPR inferences. In the proof presented here the crucial experiment is the one whose results are in Table I. According to quantum mechanics, all the runs of this experiment yield results that are in contradiction with the EPR inferences.

The fact that the contradiction occurs in all the runs resembles the four-particle version of the proof of Bell's theorem by GHZ [8,11. However, GHZ's proof is not based on the state (2) but in a "GHZ state" of four qubits. The main difference is that while in the GHZ argument, any EPR inference on one qubit requires a measurement 
on the other three qubits; in our argument, the EPR inferences on Alice's qubits require only measurements on Bob's qubits, that is, while GHZ need to consider four (or three, in the three-particle version 9 11) different combinations of two spacelike separated regions, the argument presented here needs only two spacelike separated regions.

Let us now prove properties (7)-(11). For instance, property $(7)$ is true if and only if

$$
P_{\psi}\left(A_{1} A_{3}=+1\right)>0
$$

and

$$
P_{\psi}\left(A_{1} A_{3}=+1, B_{2}=-B_{4}\right)=0 .
$$

Condition 21) is fulfilled because $P_{\psi}\left(A_{1} A_{3}=+1\right)=\frac{1}{2}$. Condition (22) is also fulfilled because the left hand side of Eq. 22) can be written as

$$
\begin{aligned}
P_{\psi}\left(A_{1} A_{3}\right. & \left.=+1, B_{2}=+1, B_{4}=-1\right)+ \\
P_{\psi}\left(A_{1} A_{3}\right. & \left.=+1, B_{2}=-1, B_{4}=+1\right)= \\
P_{\psi}\left(A_{1}\right. & \left.=+1, A_{3}=+1, B_{2}=+1, B_{4}=-1\right)+ \\
P_{\psi}\left(A_{1}\right. & \left.=-1, A_{3}=-1, B_{2}=+1, B_{4}=-1\right)+ \\
P_{\psi}\left(A_{1}=+1, A_{3}\right. & \left.=+1, B_{2}=-1, B_{4}=+1\right)+ \\
P_{\psi}\left(A_{1}=-1, A_{3}\right. & \left.=-1, B_{2}=-1, B_{4}=+1\right),
\end{aligned}
$$

where the four probabilities appearing at the right hand side of Eq. (23) are zero, due to the properties (3) and (5). Similar arguments allow us to prove properties (8)-(10).

To demonstrate property (11), it will be useful to calculate the set of common vectors of $A_{1} A_{3}$ and $a_{1} a_{3}$, and the set of common vectors of $B_{2} b_{4}$ and $b_{2} B_{4}$. The common eigenvectors of $\sigma_{z} \otimes \sigma_{z}$ and $\sigma_{x} \otimes \sigma_{x}$ are the Bell states

$$
\begin{aligned}
\left|\phi^{ \pm}\right\rangle & =\frac{1}{\sqrt{2}}(|00\rangle \pm|11\rangle), \\
\left|\psi^{ \pm}\right\rangle & =\frac{1}{\sqrt{2}}(|01\rangle \pm|10\rangle) .
\end{aligned}
$$

These Bell states satisfy the following equations:

$$
\begin{aligned}
\sigma_{z} \otimes \sigma_{z}\left|\phi^{ \pm}\right\rangle & =\left|\phi^{ \pm}\right\rangle, \\
\sigma_{x} \otimes \sigma_{x}\left|\phi^{ \pm}\right\rangle & = \pm\left|\phi^{ \pm}\right\rangle, \\
\sigma_{z} \otimes \sigma_{z}\left|\psi^{ \pm}\right\rangle & =-\left|\psi^{ \pm}\right\rangle, \\
\sigma_{x} \otimes \sigma_{x}\left|\psi^{ \pm}\right\rangle & = \pm\left|\psi^{ \pm}\right\rangle .
\end{aligned}
$$

The common eigenvectors of $\sigma_{z} \otimes \sigma_{x}$ and $\sigma_{x} \otimes \sigma_{z}$ are the Bell states

$$
\begin{aligned}
\left|\chi^{ \pm}\right\rangle & =\frac{1}{\sqrt{2}}(|0 \overline{0}\rangle \pm|1 \overline{1}\rangle), \\
\left|\omega^{ \pm}\right\rangle & =\frac{1}{\sqrt{2}}(|1 \overline{0}\rangle \pm|0 \overline{1}\rangle),
\end{aligned}
$$

where $\sigma_{x}|\overline{0}\rangle=|\overline{0}\rangle$ and $\sigma_{x}|\overline{1}\rangle=-|\overline{1}\rangle$. These Bell states satisfy the following equations:

$$
\begin{aligned}
\sigma_{z} \otimes \sigma_{x}\left|\chi^{ \pm}\right\rangle & =\left|\chi^{ \pm}\right\rangle, \\
\sigma_{x} \otimes \sigma_{z}\left|\chi^{ \pm}\right\rangle & = \pm\left|\chi^{ \pm}\right\rangle, \\
\sigma_{z} \otimes \sigma_{x}\left|\omega^{ \pm}\right\rangle & =-\left|\omega^{ \pm}\right\rangle, \\
\sigma_{x} \otimes \sigma_{z}\left|\omega^{ \pm}\right\rangle & = \pm\left|\omega^{ \pm}\right\rangle .
\end{aligned}
$$

Therefore, the probability appearing in Eq. (11) can be calculated as the probability of finding the common eigenvector of $\sigma_{z 1} \otimes \sigma_{z 3}, \sigma_{x 1} \otimes \sigma_{x 3}, \sigma_{z 2} \otimes \sigma_{x 4}$, and $\sigma_{x 2} \otimes \sigma_{z 4}$, with eigenvalues $+1,+1,+1$, and -1 , respectively; that is

$$
P=\left|\left\langle\phi^{+} \chi^{-} \mid \psi\right\rangle_{1324}\right|^{2},
$$

where $\left\langle\phi^{+} \chi^{-}\right|=\left\langle\left.\phi^{+}\right|_{13} \otimes\left\langle\left.\chi^{-}\right|_{24} \text {, and } \mid \psi\right\rangle_{1324}\right.$ is the state defined in (2), after permuting qubits 2 and 3 . To calculate $P$ it is useful to express the state (2) as

$$
\begin{aligned}
|\psi\rangle_{1324}= & \frac{1}{2 \sqrt{2}}\left(\left|\phi^{+} \chi^{-}\right\rangle+\left|\phi^{+} \omega^{+}\right\rangle-\left|\phi^{-} \chi^{+}\right\rangle\right. \\
& +\left|\phi^{-} \omega^{-}\right\rangle-\left|\psi^{+} \chi^{+}\right\rangle-\left|\psi^{+} \omega^{-}\right\rangle \\
& \left.+\left|\psi^{-} \chi^{-}\right\rangle-\left|\psi^{-} \omega^{+}\right\rangle\right) .
\end{aligned}
$$

Then is easy to obtain that $P=\frac{1}{8}$. A similar reasoning leads to each of the probabilities appearing in Table I.

These probabilities can also be obtained by realizing that a measurement of $A_{1} A_{3}$ and $a_{1} a_{3}$ is equivalent to a measurement of the Bell operator whose eigenvectors are the Bell states $\left\{\left|\phi^{ \pm}\right\rangle,\left|\psi^{ \pm}\right\rangle\right\}$on particles 1 and 3. Such measurement induces, via "entanglement swapping" [24], that particles 2 and 4 collapse to the same Bell state as particles 1 and 3 . For instance, Alice cannot obtain $A_{1} A_{3}=+1$ and $a_{1} a_{3}=+1$ (which is equivalent to obtaining $\left.\left|\phi^{+}\right\rangle_{13}\right)$ while Bob obtains $B_{2} b_{4}=+1$ and $b_{2} B_{4}=+1$ (which is equivalent to obtaining $\left|\chi^{+}\right\rangle_{24}$ ), because $\left|\phi^{+}\right\rangle_{24}$ (the state induced by entanglement swapping) is orthogonal to $\left|\chi^{+}\right\rangle_{24}$ 25.

The proof of Bell's theorem presented here can be translated into real experiments in the same way as Hardy's proof can. An experiment to test Hardy's proof consists in preparing a source of the required states and performing several tests of the required properties 23,26,27. The same strategy applies here. The source must prepare four qubits in the state (2), and we must then test properties (3)-(11) separately. From a theoretical point of view, the only difficulty lies on testing property (11), since it involves a joint measurement of $A_{1} A_{3}$ and $a_{1} a_{3}$, and a joint measurement of $B_{2} b_{4}$ and $b_{2} B_{4}$. However, as seen above, a joint measurement of $A_{1} A_{3}$ and $a_{1} a_{3}\left(B_{2} b_{4}\right.$ and $\left.b_{2} B_{4}\right)$ is equivalent to measuring a Bell operator whose eigenvectors are the Bell states $\left\{\left|\phi^{ \pm}\right\rangle,\left|\psi^{ \pm}\right\rangle\right\}\left(\left\{\left|\chi^{ \pm}\right\rangle,\left|\omega^{ \pm}\right\rangle\right\}\right)$. Indeed, since no complete discrimination between the four Bell states is needed to obtain an event which cannot be explained with local 
models (it is enough to detect, for instance, $\left|\phi^{+}\right\rangle_{13}$ and $\left.\left|\chi^{-}\right\rangle_{24}\right)$, then previous set-ups to distinguish between two of the four Bell states in the case of photons entangled in polarization 28 29], can be used for this purpose.

If a complete discrimination between the four Bell states $\left\{\left|\phi^{ \pm}\right\rangle,\left|\psi^{ \pm}\right\rangle\right\} \quad\left(\left\{\left|\chi^{ \pm}\right\rangle,\left|\omega^{ \pm}\right\rangle\right\}\right)$were possible (for some proposals, see [30 33]), it would be interesting to experimentally verify the predictions of quantum mechanics contained in Table I. That is, to verify whether, out of the 16 possible results of the experiment in which Alice measures $A_{1} A_{3}$ and $a_{1} a_{3}$, and Bob measures $B_{2} b_{4}$ and $b_{2} B_{4}$ on the state given by Eq. (2), only those eight that are unexplainable with local models have a nonzero probability to occur.

I thank David Mermin and Asher Peres for their stimulating comments on an earlier version and their valuable suggestions for its improvement.

[1] J.S. Bell, Physics (Long Island City, NY) 1, 195 (1964).

[2] L. Hardy, Phys. Rev. Lett. 71, 1665 (1993).

[3] N.D. Mermin, in Fundamental Problems in Quantum Theory, Ann. N.Y. Acad. Sci. 755, 616 (1995).

[4] G. Kar, Phys. Lett. A 204, 99 (1995).

[5] S.L. Braunstein, A. Mann, and M. Revzen, Phys. Rev. Lett. 68, 3259 (1992).

[6] R.K. Clifton and P. Niemann, Phys. Lett. A 166, 177 (1992).

[7] A. Cabello, Phys. Rev. A 61, 022119 (2000).

[8] D.M. Greenberger, M.A. Horne, and A. Zeilinger, in Bell's Theorem, Quantum Theory, and Conceptions of the Universe, edited by M. Kafatos (Kluwer, Dordrecht, 1989), p. 69.

[9] N.D. Mermin, Phys. Today 43(6), 9 (1990).

[10] N.D. Mermin, Am. J. Phys. 58, 731 (1990).

[11] D.M. Greenberger, M.A. Horne, A. Shimony, and A. Zeilinger, Am. J. Phys. 58, 1131 (1990).

[12] S. Goldstein, Phys. Rev. Lett. 72, 1951 (1994).

[13] N.D. Mermin, Am. J. Phys. 62, 880 (1994).

[14] S. Kochen and E.P. Specker, J. Math. Mech. 17, 59 (1967).

[15] A. Peres, Phys. Lett. A 151, 107 (1990).

[16] N.D. Mermin, Phys. Rev. Lett. 65, 3373 (1990).

[17] A. Peres, Found. Phys. 22, 357 (1992).

[18] N.D. Mermin, in Group Theoretical Methods in Physics. Proc. of the XIX International Colloquium (Salamanca, Spain, 1992), edited by M.A. del Olmo, M. Santander, and J. Mateos Guilarte (Ciemat-Real Sociedad Española de Física, Madrid, 1993), Vol. 2, p. 3; Rev. Mod. Phys. 65, 803 (1993).

[19] A. Peres, Quantum Theory: Concepts and Methods (Kluwer, Dordrecht, 1993), p. 151.

[20] A. Peres, Found. Phys. 26, 807 (1996).

[21] A. Einstein, B. Podolsky, and N. Rosen, Phys. Rev. 47, 777 (1935).
[22] L. Hardy, in New Developments on Fundamental Problems in Quantum Physics, edited by M. Ferrero and A. van der Merwe (Kluwer, Dordrecht, 1997), p. 163.

[23] D. Boschi, S. Branca, F. De Martini, and L. Hardy, Phys. Rev. Lett. 79, 2755 (1997).

[24] C.H. Bennett, G. Brassard, C. Crépeau, R. Jozsa, A. Peres, and W.K. Wootters, Phys. Rev. Lett. 70, 1895 (1993).

[25] As an aside, the mutually orthogonal states $\left|\phi^{+}\right\rangle,\left|\psi^{-}\right\rangle$, $\left|\chi^{+}\right\rangle$, and $\left|\omega^{-}\right\rangle$play a central role in the experiment of the Kochen-Specker theorem proposed in A. Cabello and G. García Alcaine, Phys. Rev. Lett. 80, 1797 (1998).

[26] J.R. Torgerson, D. Branning, C.H. Monken, and L. Mandel, Phys. Lett. A 204, 323 (1995).

[27] D. Boschi, F. De Martini, and G. Di Giuseppe, Phys. Lett. A 228, 208 (1997); G. Di Giuseppe, F. De Martini, and D. Boschi, Phys. Rev. A 56, 176 (1997).

[28] K. Mattle, H. Weinfurter, P.G. Kwiat, and A. Zeilinger, Phys. Rev. Lett. 76, 4656 (1996).

[29] D. Bouwmeester, J-W. Pan, K. Mattle, M. Eibl, H. Weinfurter, and A. Zeilinger, Nature (London) 390, 575 (1997).

[30] M.O. Scully, B.-G. Englert, and C.J. Bednar, Phys. Rev. Lett. 83, 4433 (1999).

[31] E. DelRe, B. Crosignani, and P. Di Porto, Phys. Rev. Lett. 84, 2989 (2000).

[32] D. Vitali, M. Fortunato, and P. Tombesi, Phys. Rev. Lett. 85, 445 (2000).

[33] M.G.A. Paris, M.B. Plenio, S. Bose, D. Jonathan, and G.M. D'Ariano, Phys. Lett. A 273, 153 (2000).

\begin{tabular}{ccccc}
\hline \hline$A_{1} A_{3}$ & $a_{1} a_{3}$ & $B_{2} b_{4}$ & $b_{2} B_{4}$ & Probability \\
\hline+1 & +1 & \pm 1 & \pm 1 & 0 \\
+1 & +1 & \pm 1 & $\mp 1$ & 0.125 \\
+1 & -1 & \pm 1 & \pm 1 & 0.125 \\
+1 & -1 & \pm 1 & $\mp 1$ & 0 \\
-1 & +1 & \pm 1 & \pm 1 & 0.125 \\
-1 & +1 & \pm 1 & $\mp 1$ & 0 \\
-1 & -1 & \pm 1 & \pm 1 & 0 \\
-1 & -1 & \pm 1 & $\mp 1$ & 0.125 \\
\hline \hline
\end{tabular}

TABLE I. Probabilities of the 16 possible results of the experiment in which Alice measures $A_{1} A_{3}$ and $a_{1} a_{3}$ and Bob measures $B_{2} b_{4}$ and $b_{2} B_{4}$ on the state given by Eq. (2). 